\title{
Homocysteine, Vitamin B12 and Folic Acid in Children with Acute Glomerulonephritis
}

\author{
Duangkamol Viroonudomphol ${ }^{1}$, Saowanee Kanjanachumpol ${ }^{2}$, Sarinya Sirisate ${ }^{3}$ \\ ${ }^{1}$ Faculty of Nursing, Siam University, Bangkok, Thailand \\ ${ }^{2}$ Faculty of Medicine Ramathibody Hospital, Mahidol University, Bangkok, Thailand \\ ${ }^{3}$ Department of Pediatrics, Faculty of Medicine Ramathibody Hospital, Mahidol University, Bangkok, Thailand \\ Email: v_duangkamol@yahoo.com
}

How to cite this paper: Viroonudomphol, D., Kanjanachumpol, S. and Sirisate, S. (2018) Homocysteine, Vitamin B12 and Folic Acid in Children with Acute Glomerulonephritis. World Journal of Engineering and Technology, 6, 661-670.

https://doi.org/10.4236/wjet.2018.63042

Received: July 12, 2018

Accepted: August 7, 2018

Published: August 10, 2018

Copyright $\odot 2018$ by authors and Scientific Research Publishing Inc. This work is licensed under the Creative Commons Attribution International License (CC BY 4.0).

http://creativecommons.org/licenses/by/4.0/

(c) (i) Open Access

\begin{abstract}
Homocysteine (Hcy) is an intermediate product of methionine formed by its demethylation. Hcy can be metabolized via remethylation to methionine or transsulfuration to cysteine which is dependent on several enzymes and cofactors. It is deleterious to blood vessel including glomeruli. Kidney is a major organ that metabolizes Hcy. More than $80 \%$ of patients with chronic renal disease develop hyperhomocysteinemia (hHcy). Accessible data of plasma Hcy in nephritic syndrome (NS) patients are controversial with increased, decreased and unchanged values reported. In renal patients, plasma Hcy concentration can be reduced by administration of folic acid. Absolute or relative deficiencies of folate, vitamin B6, or vitamin B12 may also play a role. Therefore, plasma Hcy, folic acid, vitamin B6, and vitamin B12 in children with acute glomerulonephritis (AGN) were accessed in this study. Hcy, folic acid vitamin B12, B6 and renal function such as blood urea nitrogen (BUN), creatinine ( $\mathrm{Cr}$ ) were analyzed 12 pediatric patients with AGN and 15 age and sex matched healthy children served as controls. The results revealed that a significant increase in plasma Hcy in children with acute AGN when compared with controls. For simple regression analysis, Hcy was positively correlated with BUN, Cr, ferritin and uric acid but negatively correlated with serum glutathione. This research indicated hHcy suggests enhanced risks for inflammation and endothelial injury, which lead to kidney disease. Folic acid has also been shown to improve endothelial function, suggesting an alternative explanation for the effect of folic acid on endothelial function. Careful considerations of not only dietary measures are necessary but also folate and vitamin $\mathrm{B}$ supplementation for reducing hHcy in AGN need to be investigated.
\end{abstract}

\section{Keywords}

Folic Acid, Homocysteine, Hyperhomocysteinemia, Acute Glomerulonephritis 


\section{Introduction}

Homocysteine (Hcy) is an intermediate product of methionine formed by its demethylation. Hcy can be metabolized via remethylation to methionine or transsulfuration to cysteine which is dependent on several enzymes and cofactors. Hcy is metabolized in the liver and kidney. Both organs process the essential Hcy-metabolizing enzymes. Normal kidney plays an important role in maintaining the level of Hcy in the circulation through filtration. Hyperhomocysteinemia (hHcy) refers to an elevated circulating level of the sulfur-containing amino acid hHcy and has been shown to be a risk factor for vascular disease in the general population. Hcy level increases as renal function declines and processes to end-stage renal disease (ESRD) with the majority ( $>85 \%)$ of patients ultimately experiencing mind-to-moderate hHcy [1]. Numerous studies confirm the strong and strikingly consistent inverse relationship between Hcy levels and renal function over a broad range of glomerular filtration rates $(60 \mathrm{ml}$ to 165 $\mathrm{ml} / \mathrm{min}$ ). Data obtained from the United States third National Health and Nutrition Survey between 1991-1994 showed that among adults with moderate to severe loss of renal function from creatinine clearance (Ccr) calculated from the Cockroft-Gault formula, the odd ratio (OR) for elevated Hcy associated with Ccr $\leq 50 \mathrm{ml} / \mathrm{min} / 1.73 \mathrm{~m}^{2}$ compared to $\mathrm{Ccr}>80 \mathrm{ml} / \mathrm{min} / 1.73 \mathrm{~m}^{2}$ was 9.1 in men and 2.9 in women. With marginally subnormal renal function (Ccr 51 - 80 $\mathrm{ml} / \mathrm{min} / 1.73 \mathrm{~m}^{2}$ ) compared to normal renal function (Ccr $>80 \mathrm{ml} / \mathrm{min} / 1.73 \mathrm{~m}^{2}$ ), the ORs were 1.3 in men and 1.2 in women [2].

Kidney is a major organ that metabolizes Hcy. More than $80 \%$ of patients with chronic renal disease develop hyperhomocysteinemia (hHcy). This result can be ascribed to a decrease in intrarenal Hcy clearance and/or decrease in extrarenal Hcy clearance, perhaps attributable to retained uremic toxins that inhibit Hcy metabolism. In chronic renal disease, remethylation is diminished while transsulfuration tends to be reduced or unchanged [3] [4] [5]. The similarity in the pathology of atherosclerosis and glomerulosclerosis is well recognized. Both are associated with influx of monocytes, production of lipid-laden macrophages, presence of cholesterol and cholesterol ester, proliferation of vascular smooth muscle cells, intima thickening, matrix expansion, elastic lamina disruption, and luminal platelet accumulation [6] [7]. Due to the similarity in the pathogen between atherosclerosis and glomerulosclerosis and to the similarity in the pathology between atherosclerosis and glomerulosclerosis that Hcy is an essential risk factor for atherosclerosis, it is logical to speculate that hHcy may lead to progressive kidney injury and glomerulosclerosis, and the normalization of high plasma Hcy may retard progression of chronic renal disease. Publication of plasma Hcy in chronic renal disease appeared for the first time in adults in 1979 [8]. In contrast, it appeared for the first time in children 20 years later in 1999 [9].

In addition inflammation commonly seen in chronic kidney disease (CKD) patients has been shown to cause low plasma amino acid concentrations [10]. 
Many characteristic alterations in the plasma amino acid profile that are observed in chronic end-stage renal disease (CESRD) are already present in mind renal in sufficiency. Progressive loss of renal function generally results in increasing abnormalities, these changes in plasma amino acids were usually linear with reduction in glomerular filtration rate (GFR) [11]. Plasma protein and amino acid concentrations have been reported to be abnormal in patients with CRF, whether on conservative or regular dialysis treatment [12] [13] [14]. Hcy is thrombogenic as it increases thromboxane formation antagonizes nitric oxide, enhances platelet aggregation, and inhibits protein $\mathrm{C}$ and thrombomodulin. Hcy is also potent mitogen for vascular nsmooth muscle cells [15] [16].

Therefore, in end-stage renal disease, elevated plasma Hcy concentrations could contribute to the high prevalence of cardiovascular disease and increased mortality rate [16]. HHcy at an earlier stage could also accelerate progression of chronic renal disease [17]. The present study was to not only determine Hcy concentration, vitamin B12 and folic acid in children with AGN but also determine the relationship between plasma Hcy and clinical parameter (blood pressure, proteinuria), renal function test (BUN, $\mathrm{Cr}$ ).

\section{Objectives}

1) To determine plasma Hcy, vitamin B12 and folic acid at interval in children with AGN.

2) To determine the association between plasma Hcy and clinical parameter (blood pressure, proteinuria), renal function test (BUN, Cr), inflammation (ferritin) and antioxidant (uric acid and glutathione).

\section{Materials and Method}

Protocol was approved by the Ethics Committee of the faculty of medicine Ramathibody hospital. An informed consent had got from parents of all participants before the study was performed. The sample size was calculated based on a $80 \%$ power to detect a $30 \%$ increase of plasma Hcy in AGN with a error $=0.05$, and dropout rate $=20 \%$. The number of AGN patients $=22$ and controls $=22$. The subjects in this study consisted of participants 12 pediatric patients with AGN who attended at the Paediatric Nephrology Clinic of Ramathibody Hospital, and 15 age and sex-matched healthy children who were from a school in Bangkok served as controls. Inclusion criteria was children ages up to 16 years old with acute onset of primary glomerular disease defined as sudden onset of hematuria, edema, hypertension and renal insufficiency. For exclusion criteria followed as chronic renal disease, secondary glomerular disease and patients withdraw from the study. Venous blood was taken from each subject in the morning, after an overnight fast from patients at 0, 3, 7, 28 and $\geq 60$ days after admission. For each control a once blood sample was taken for determination of biochemical parameters.

Determination of plasma Hcy concentrations used commercial automated 
fluorescence polarizations of immunoassay (FPIA) system, the Abbott Hcy assay kit with the Abbott analyzer (Abbott Laboratories, Abbott Park, IL). The serum concentrations of BUN and creatinine $(\mathrm{Cr})$ were determined with routine methods. The serum folic acid, RBC folate and vitamin B12 were analyzed using Vitros Immunodiagnostic System (Ortho Clinical Diagnostics, Amersham, U.K.).

\section{Statistical Analysis}

Data were expressed as mean $\pm \mathrm{SD}$ or median (minimum, maximum) depended on the type of data distribution. Unpair t-test or Mann-Whitney $U$ test was used for comparison between two groups. Simple regression analysis was used for the relationships between continuous variables. All tests were two-tailed, and a $p$ value of less than 0.05 was considered to be statistically significant.

\section{Results}

There were 14 AGN patients but 2 of them turned out to be SLE and they were excluded. 15 controls age and sex matched schoolchildren. The clinical characteristics of the $12 \mathrm{AGN}$ patients and the 15 controls were shown in Table 1. All AGN had poteinuria and hematuria; 9 out of 11 had increased antistreptolysin $O$ (ASO) titer (>150 IU/ml) and decreased concentration of serum complement (C3) $(<900 \mu \mathrm{g} / \mathrm{ml})$. Systolic blood pressure (SBP) was higher in the patients than in the controls, but diastolic blood pressure was not different between the two groups.

Results of plasma Hcy, BUN and Cr were as in Tables 2-4 respectively. Hcy and BUN at day 0 were significantly higher in the patients than controls but concentrations of $\mathrm{Cr}$ at day were not different between 2 groups. In the patients group, $\mathrm{Hcy}, \mathrm{BUN}$ and $\mathrm{Cr}$ significantly decreased with time; all decreased to normal values by day 28 .

Mean value of serum vitamin B12, serum folic acid, RBC folic acid and plasma vitamin B6 were shown in Table 5. There were no differences in serum vitamin B12 and serum folic acid at day 0 between 2 groups. The values of RBC folic acid were significantly.

The results of urine analysis (proteinuria and microscopic hematuria) were shown in Table 6. The findings were gradual decline with time in the AGN patients.

Correlation between Hcy and clinical parameters and renal function were presented in Table 7. Significantly positive correlations were found between plasma Hcy and respectively, SBP, DBP, proteinuria, and in particular, BUN and Cr.

\section{Discussion}

The results in this study have shown for the first time that all children with AGN had high plasma levels of Hcy and RBC folic acid, low serum levels of vitamin B6 in parallel to the values of BUN and Cr. The plasma Hcy levels increased about 
Table 1. Clinical characteristics of children with AGN and controls expressed as mean \pm SD or median (min, max).

\begin{tabular}{cccc}
\hline Parameter & $\begin{array}{c}\text { AGN } \\
(\mathrm{n}=12)\end{array}$ & $\begin{array}{c}\text { Controls } \\
(\mathrm{n}=15)\end{array}$ & $\mathrm{p}$ \\
\hline Age $(\mathrm{Yr})$ & $11.0 \pm 3.5$ & $12.1 \pm 3.3$ & 0.736 \\
Sex $(\mathrm{M} / \mathrm{F})$ & $3 / 9$ & $5 / 10$ & 0.419 \\
BMI $\left(\mathrm{kg} / \mathrm{m}^{2}\right)$ & $17.69 \pm 4.68$ & $18.20 \pm 3.45$ & $0.014^{*}$ \\
SBP $(\mathrm{mmHg})$ & $129.5 \pm 19.3$ & $112.6 \pm 14.1$ & 0.300 \\
DBP $(\mathrm{mmHg})$ & $81.5 \pm 11.8$ & $76.5 \pm 12.7$ & \\
Proteinuria & positive & Not done & \\
Hematuria & positive & Not done & \\
ASO $(\mathrm{IU} / \mathrm{ml})$ & $322(138.1430)$ & Not done & \\
ASO $>150 \mathrm{IU} / \mathrm{ml}$ & $9 / 11$ & Not done & \\
C3 $(\mu \mathrm{g} / \mathrm{ml})$ & $227(72.1110)$ & Not done & \\
C3 $<900 \mu \mathrm{g} / \mathrm{ml}$ & $9 / 11$ & Not done & \\
\hline
\end{tabular}

Table 2. Values of plasma Hcy in AGN and controls.

\begin{tabular}{|c|c|c|c|}
\hline \multirow{2}{*}{ Day } & \multicolumn{2}{|c|}{ Hcy $(\mu \mathrm{mol} / \mathrm{L})$} & \multirow{2}{*}{$P$ value } \\
\hline & AGN & Controls & \\
\hline 0 & $12.7 \pm 4.4$ & & 0.003 \\
\hline 3 & $13.5 \pm 3.7$ & & $<0.001$ \\
\hline 7 & $13.1 \pm 2.8$ & $8.4 \pm 2.4^{\mathrm{a}}$ & $<0.001$ \\
\hline 28 & $9.4 \pm 3.3$ & & 0.407 \\
\hline$\geq 60$ & $7.3 \pm 2.2$ & & 0.250 \\
\hline
\end{tabular}

$P$ value $^{\mathrm{c}}<0.001$

${ }^{a}$ obtain once. ${ }^{\mathrm{b}}$ comparison between AGN patients and controls. ${ }^{\mathrm{c}}$ change with time in AGN patients.

Table 3. Values of BUN in AGN and controls.

\begin{tabular}{|c|c|c|c|}
\hline \multirow{2}{*}{ Day } & \multicolumn{2}{|c|}{ BUN (mg/dL) } & \multirow{2}{*}{$P$ value } \\
\hline & AGN & Controls & \\
\hline 0 & $21.0(12.49)$ & & $<0.001$ \\
\hline 3 & $22.0(12.37)$ & & $<0.001$ \\
\hline 7 & $20.0(11.35)$ & $11.0(9.16)^{\mathrm{a}}$ & 0.002 \\
\hline 28 & $14.0(7.19)$ & & 0.032 \\
\hline$\geq 60$ & $11.0(7.14)$ & & 0.712 \\
\hline
\end{tabular}

$P$ value ${ }^{\mathrm{c}}<0.003$

${ }^{a}$ obtain once. ${ }^{b}$ comparison between AGN and controls. ${ }^{c}$ change in creatinine with time in patients. 
Table 4. Values of $\mathrm{Cr}$ in AGN and controls.

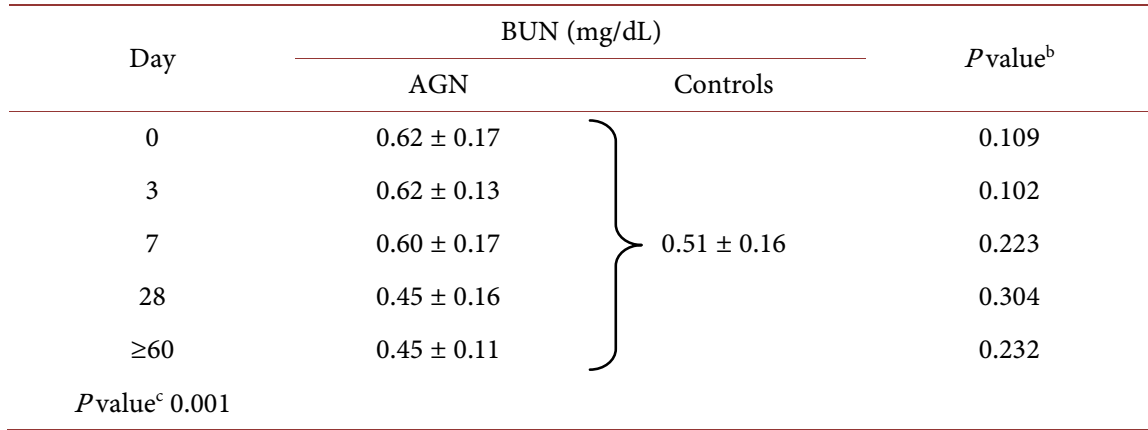

a obtain once. ${ }^{b}$ comparison between AGN and controls. ${ }^{\mathrm{c}}$ change in creatinine with time in patients.

Table 5. Value of vitamin B6, B12 and folic acid in AGN and controls.

\begin{tabular}{ccccc}
\hline Parameter & Subject & Day 0 & Day 28 & $P$ \\
\hline Plasma B6 & AGN & $25.6 \pm 13.5$ & & \\
$(\mathrm{mmol} / \mathrm{l})$ & controls & $53.6 \pm 7.8$ & $34.6 \pm 11.7$ & 0.126 \\
& $p$ & $<0.001$ & & 0.878 \\
Serum B12 & AGN & $576 \pm 176$ & & \\
$(\mathrm{pmol} / \mathrm{l})$ & controls & $502 \pm 169$ & $562 \pm 234$ & \\
& $p$ & 0.281 & & 0.730 \\
Serum folic acid & AGN & $13.4 \pm 4.3$ & & \\
$(\mathrm{mmol} / \mathrm{l})$ & controls & $13.6 \pm 4.1$ & $12.9 \pm 3.9$ & \\
\end{tabular}

Table 6. Median (min, max) of urine protein and urine RBC in AGN patients.

\begin{tabular}{ccc}
\hline Day & Urine protein $(+)$ & Urine red blood cell per low-power field \\
\hline 0 & $3(1.4)$ & $35(5.200)$ \\
3 & $2(0.4)$ & $75(3.200)$ \\
7 & $2(0.4)$ & $35(3.200)$ \\
28 & $1(0.3)$ & $50(5.100)$ \\
$\geq 60$ & $0(0.4)$ & $20(3.100)$ \\
$p$ value $^{*}$ & 0.008 & 0.068 \\
\hline
\end{tabular}

${ }^{*}$ Change in urine protein and rbc with time in AGN patients.

Table 7. Correlations between Hcy and clinical parameters, renal function, ferritin, uric acid and glutathione.

\begin{tabular}{lccc}
\hline \multicolumn{1}{c}{ Variables } & $\mathrm{r}$ & $\mathrm{R}^{2}$ & $P$ \\
Clinical parameters: & & & 0.001 \\
-SBP & 0.454 & 0.206 & 0.005 \\
-DBP & 0.391 & 0.153 & 0.006 \\
-Proteinuria & 0.365 & 0.133 & \\
Renal function: & & & $<0.001$ \\
-BUN & 0.700 & 0.490 & $<0.001$ \\
-Cr & 0.808 & 0.625 & 0.015 \\
Inflammatory & & & $<0.001$ \\
-Ferritin & 0.369 & 0.136 & 0.002 \\
Antioxidant & & & 0.484 \\
-Uric acid & 0.696 & 0.167 & \\
-Glutathione & -0.409 & & \\
\hline
\end{tabular}


$50 \%$ and decline to normal by day 28. The Hcy in normal children in this study was $8.5 \pm 2.5 \mu \mathrm{mol} / \mathrm{l}$ which is similar results of Hcy levels at $8.6 \pm 3.4 \mu \mathrm{mol} / \mathrm{l}$ those published in the literature [18]. There are controversies about the prevalence of hyperhomocysteine among nephrotic syndrome in previous studies. These studies showed that plasma Hcy levels were either decreased [19], equal [20] or increased [21] in nephritic syndrome (NS) patients compared to patients without NS and of similar renal function [19] [20] or to healthy controls [21]. The findings of higher level of Hcy in NS agree with the report of these previous studies [22] [23]. The observed hHcy in AGN patients is due to impaired renal function, vitamin B6, B12 and folic acid deficiencies. Vitamin B6 was significantly lower in AGN than controls. Vitamin B6 is involved in the catabolism of Hcy via the transsulfuration pathway of Hcy. Whether the reduction of vitamin B6 decreased the transsulfuration (catabolism) of Hcy, thereby increasing plasma Hcy remains to be elucidated. Recently, Yi and Li have reviewed and proposed mechanisms of Hcy-induced glomerular injury [24], one mechanism involves local oxidative stress and inflammatory factors. Oxidative stress results from an imbalance between oxidation and antioxidation. In this study, one of antioxidants, glutathione, was markedly reduced in the patients [25]. This study has also shown that both folic and vitamin B6 levels were lower in the AGN than healthy controls. These are similar findings of other studies in children [26] [27]. Reduced nutritional intakes of vitamins and possibly other micronutritents in these children may explain the decrease in serum concentration of these essential vitamins [28]. These may be indicated the adequate folate and vitamin Bs in children's diet and could be resolved by increasing vegetables and fruit consumption, both good source of folate, which will reduce homocysteine levels and increase folate levels as presented in previous study [29]. However, considering the health implications of low levels of serum folate, vitamin B12 and vitamin B6, it is important that adequate insights are gained into the import and reasons for low vitamins in patients with NS. No differences in the C677T and A1298C MTHFR genotype deficiencies were found between the AGN and controls in this study (data not shown). The limitation that needs to be considered of data presentation in this study is the sample size.

\section{Conclusion}

Clinical important hyperhomocysteinemia and low vitamins B were demonstrated for the early in children with AGN. The concentrations of plasma Hcy paralleled with BUN and serum Cr. There were apparent associations between Hcy and oxidative stress and inflammatory markers. The results suggest that not only potential future study has the effect of folate and B vitamins therapy on hHcy levels but also has public health importance, considering the fact that cardiovascular complications are gradually becoming a common cause of death in many developing countries especially among individual with renal disease [30]. 


\section{Conflicts of Interest}

The authors declare no conflicts of interest regarding the publication of this paper.

\section{References}

[1] Kang, S.S., Wong, P.W., Susmano, A., et al. (1991) Thermoabile Methylenetetrahydrofolate Reductase: An Inherited Risk Factor for Coronary Artery Disease. American Journal of Human Genetics, 48, 536-545.

[2] Foley, R.N., Parfrey, P.S. and Sarnak, M.J. (1998) Clinical Epidemiology of Cardiovascular Disease in Chronic Renal Disease. American Journal of Kidney Disease, 32, S112-S119. https://doi.org/10.1053/ajkd.1998.v32.pm9820470

[3] Guttormsen, A.B., Ueland, P.M., Svastad, E., et al. (1997) Kinetic Basis of Hyperhomocysteinemia in Patients with Chronic Renal Failure. Kidney International, 52, 495-502. https://doi.org/10.1038/ki.1997.359

[4] Van Guldener, C., Kulik, W., Berger, R., et al. (1999) Homocysteine and Methionine Metabolism in ESRD: A Stable Isotope Study. Kidney International, 56, 1064-1071. https://doi.org/10.1046/j.1523-1755.1999.00624.x

[5] Stam, F., van Guldener, C., ter Wee, P.M., et al. (2004) Homocysteine Clearance and Methylation Flux Rates in Healthy and End-Stage Renal Disease: Association with S-Adenosylhomocysteine. American Journal of Physiology-Renal Physiology, 287, F215-F223. https://doi.org/10.1152/ajprenal.00376.2003

[6] Chen, P., Poddar, R., Tipa, E.V., et al. (1999) Homocysteine Metabolism in Cardiovascular Cells and Tissues: Implication for Hyperhomocysteinemia and Cardiovascular Disease. Advances in Enzyme Regulation, 39, 93-109. https://doi.org/10.1016/S0065-2571(98)00029-6

[7] Diamond, J.R. (1991) Analogous Pathobiologic Mechanism in Glomerulosclerosis and Atherosclerosis. Kidney International, 31, S29-S34.

[8] Keane, W.F., Kasiske, B.L. and O’Donnell, M.P. (1988) Lipids and Progressive Glomerulosclerosis. A Model Analogous to Atherosclerosis. American Journal of Nephrology, 8, 261-271. https://doi.org/10.1159/000167599

[9] Jacques, P.F., Selhub, J., Bostom, A.G., et al. (1999) The Effect of Folic Acid Fortification on Plasma Folate and Total Homocysteine Concentrations. New England Journal of Medicine, 340, 1449-1454. https://doi.org/10.1056/NEJM199905133401901

[10] Suliman, M.E., Qureshi, A.R., Stenvinkel, P., et al. (2005) Inflammation Contributes to Low Plasma Amino Acid Concentrations in Patients with Chronic Kidney Disease. The American Journal of Clinical Nutrition, 82, 342-349.

https://doi.org/10.1093/ajcn/82.2.342

[11] Sawy, M.A., Zaki, M.M., El-Hakim, I.L., Mowafy, M.E., et al. (2012) Serum Amino Acid Abnormalities in Mind Renal Insufficiency Patients with Chronic Renal Failure with and without History of Thromboebolic Manifestations. The Egyptian Journal of Medical Human Genetics, 13, 73-80. https://doi.org/10.1016/j.ejmhg.2011.06.007

[12] Saifan, C., EI-Chalabaty and EI-Sayegh, S. (2013) Hyperhomocysteinemia and Vascular Access Thrombosis in Hemodialysis Patients: A Retrospective Study. Vascular Health and Risk Management, 9, 361-364. https://doi.org/10.2147/VHRM.S47255

[13] Suliman, M.E., Divino Filho, J.C., Barany, P., Anderstam, B., et al. (1999) Effect of High Dose Folic Acid and Pyridoxine on Plasma and Erythrocyte Sulfur Amino 
Acid in Hemodialysis Patients. Journal of American Society of Nephrology, 10, 1287-1296.

[14] Suliman, M.E., Stevinkel, P., Heibuger, O., Barany, P., et al. (2002) Plasma Sulfur Amino Acids in Relation to Cardiovascular Disease, Nutritional Status, and Diabetes Mellitus in Patients with Chronic Renal Failure at Start of Dialysis Therapy. American Journal of Kidney Disease, 40, 480-488. https://doi.org/10.1053/ajkd.2002.34887

[15] Klamroth, R., Orlovic, M., Fritsche, I., Scibt, S., et al. (2013) The Influence of Thrombophillic Risk Factors on Vascular Access Survival in Chronic Dialysis Patients in a Retrospective Evaluation. Vasa, 42, 32-39. https://doi.org/10.1024/0301-1526/a000245

[16] Shakeri, A., Abdi, M., Khosrosshahi, H.T. and Fouladi, R.F. (2011) Common Carotid Artery Intema-Media Thickness and Alterosclerotic Plaque in Carotid Bulb in Patients with Chronic Kidney Disease on Hemodialysis a Case-Control Study. Pakistan Journal of Biological Sciences, 14, 844-848. https://doi.org/10.3923/pjbs.2011.844.848

[17] Aminzadeh, M.A., Gollapudi, P. and Vaziri, N.D. (2011) Effect of Nephritic Syndrome on Homocysteine Metabolism. Nephrology Dialysis Transplantation, 26, 1244-1247. https://doi.org/10.1093/ndt/gfq551

[18] Alberto, C., Alba, C., et al. (2003) Homocysteine, Folate, Vitamin B12 Levels and C677T MTHFR Mutation in Children with Renal Failure. Pediatric Nephrology, 18, 225-229.

[19] Annadottir, M., Hultberg, B. and Berg, A.L. (2001) Plasma Total Homocysteine Concentration in Nephrotic Patients with Idiopathic Membranous Nephropathy. Nephrology Dialysis Tranplantation, 16, 45-47. https://doi.org/10.1093/ndt/16.1.45

[20] Dogra, G., Irish, A.B. and Watts, G.F. (2001) Homocysteine and Nephritic Syndrome. Nephrology Dialysis Tranplantation, 16, 1720-1721. https://doi.org/10.1093/ndt/16.8.1720

[21] Joven, J., Arcelus, R., Camps, J., Ordonez-Lianos, J., et al. (2000) Determinants of Plasma Homocyst(e)ine in Patients with Nephritic Syndrome. Journal of Molecular Medicine, 78, 147-154. https://doi.org/10.1007/s001090000093

[22] Podda, G.M., Lussana, F., Moroni, G., Faioni, E.M., Lombardi, R., Fontana, G., Ponticelli, C. and Cattaneo, M. (2007) Abnormalities of Homocysteine and B Vitamins in the Nephritic Syndrome. Thrombosis Research, 120, 647-652.

https://doi.org/10.1016/j.thromres.2006.12.011

[23] Terderenda, E., Korzeniecka-Kozerska, A., Polowski, T., Wasilewska, A. and Zoch-Zwierz, W. (2011) Serum and Urinary Homocysteine in Children with Steroid-Dependent Nephritic Syndrome. Polski Merkuriusz Lekarski, 31, 204-208.

[24] Yi, F. and Li, P.-L. (2008) Mechanism of Hcy-Induced Glomerular Injury and Sclerosis. American Journal of Nephrology, 28, 254-264. https://doi.org/10.1159/000110876

[25] Meister, A. (1994) Glutathione-Ascorbic Acid Antioxidant System in Animals. The Journal of Biological Chemistry, 269, 9937-9400.

[26] Bates, C.J. and Mansoor, M.A. (2002) Correlates of Plasma Homocysteine, Cysteine and Cyateinyl-Glycine in Respondents in the British National Diet and Nutrition Survey of Young People Aged 4-18 Years, and a Comparison with the Survey of People Aged 65 Years and over. British Journal of Nutrition, 87, 71-79. https://doi.org/10.1079/BJN2001479

[27] Osganian, S.K., Stampfer, M.J., Spiegelman, D., et al. (1999) Distribution of and 
Factors Associated with Serum Homocysteine Levels in Children: Child and Adolescent Trial for Cardiovascular Health. Journal of American Medical Association, 281, 1189-1196. https://doi.org/10.1001/jama.281.13.1189

[28] Bjorke, M.A.L., Refsum, H., Markestad, T. and Ueland, P.M. (2003) Cobalamin Status and Its Biochemical Markers Methylmalonic Acid and Homocysteine in Different Age Groups from 4 Days to 19 Years. Clinical Chemistry, 49, 2067-2075. https://doi.org/10.1373/clinchem.2003.019869

[29] Stanger, O., Herrman, W., Pietrzik, K., Fowler, B., Geisel, J., Dierkes, J. and Weger, M. (2003) DACH-LIGA Homocysteine (German, Austrian and Swiss Homocysteine Society), Consensus Paper on the Rational Clinical Use of Homocysteine, Folic Acid and B-Vitamins in Cardiovascular and Thrombotic Diseases: Guidelines and Recommendations. Clinical Chemistry and Laboratory Medicine, 41, 1392-1403. https://doi.org/10.1515/CCLM.2003.214

[30] Silva, L.S., Oliveira, R.A., Silva, G.B., Lima, J.W., Silva, R.P., Liborio, A.B., Daher, E.F. and Sobrinho, C.R. (2012) Cardiovascular Disease in Patients with End-Stage Renal Disease on Hemodialysis in Developing Country. Saudi Journal of Kidney Disease and Transplantation, 23, 262-266. 